\title{
Método intervención en la reducción del índice de accidentabilidad en la contratista minera Aesa
}

\author{
Intervention method in the reduction the accidentability index in the Aesa \\ mining cntractor
}

\author{
Manuel Enrique López Montalbán ${ }^{1}$, Alfonso Alberto Romero Baylón ${ }^{2}$
}

Recibido: 01/07/2020 - Aprobado: 30/10/2020 - Publicado: 30/11/2020

\begin{abstract}
RESUMEN
La implementación del método intervención reduce el índice de accidentabilidad en la contratista minera AESA y contribuye a que se vaya creando una cultura de seguridad tanto en el trabajador como en la empresa. Se ha mejorado en muchos aspectos en el tema de seguridad. Ahora, se cuenta con normas de seguridad, procedimientos, formatos, estándares, metodologías de seguridad basadas en el comportamiento de las personas, los incidentes y otros eventos generados por la naturaleza y la roca. También se cuenta con programas de respuesta inmediata ante emergencias suscitadas. La influencia del método de intervención en la reducción del índice de accidentabilidad ha sido altamente positiva puesto que los trabajadores se han informado y capacitado en los temas de seguridad mediante la implementación de este método; ha mejorado la cultura de seguridad, puesto que ahora un $87.5 \%$ de los participantes sabe lo que es seguridad laboral, la rotación del personal ha mejorado considerablemente puesto que los trabajadores antes no sabían los beneficios de tener un seguro de vida, para mantenerse más seguros en su lugar de trabajo y evitar la rotación del personal, la reducción de la frecuencia de accidentes incapacitantes ha sido considerablemente puesto que los trabajadores han sido capacitados mediante el método sobre los beneficios que ofrece el IPERC para calificarlos según la peligrosidad.
\end{abstract}

Palabras claves: Método de intervención; accidentabilidad; minería; trabajos de riesgo.

\begin{abstract}
The implementation of the intervention method reduces the accident rate in the AESA mining contractor and contributes to the creation of a safety culture in both the worker and the company. Security has been improved in many aspects. Now, there are security norms, procedures, formats, standards, security methodologies based on the behavior of people, incidents and other events generated by nature and rock. There are also immediate response programs for emergencies that arise. The influence of the intervention method in reducing the accident rate has been highly positive since workers have been informed and trained in safety issues by implementing this method; The safety culture has improved, since now $87.5 \%$ of the participants know what job safety is, staff turnover has improved considerably since workers previously did not know the benefits of having life insurance, to stay safer in their workplace and avoid staff turnover, the reduction in the frequency of disabling accidents has been considerably since workers have been trained using the method on the benefits offered by IPERC for the identification of hazards and assess risks to qualify them according to the dangerousness.
\end{abstract}

Keywords: Supply management; quality of service; supply chain.

1 Ingeniero de Minas. Jefe de seguridad contratista minera AESA, Universidad Nacional Mayor de San Marcos. Lima, Perú. Autor para correspondencia: mlopez23a@gmail.com

2 Ingeniero de Minas. Vicedecano Académico de la Facultad de Ingeniería Geológica, Minera, Metalúrgica y Geográfica, Universidad Nacional Mayor de San Marcos. Lima, Perú. Código 0A0330. E-mail: alfonso.romero@unmsm.edu.pe ORCID: https://orcid.org/0000-0002-4433-0111 


\section{INTRODUCCIÓN}

La minería es una de las actividades que existen desde muy antiguo, desde hace aproximadamente 2,5 millones de años, cuando el hombre extraía piedras y metales para la fabricación de herramientas, fabricar utensilios primitivos mediante el tallado de rocas, consistiendo en una actividad sencilla basada en la extracción de sílex, cuyos restos o asientos mineros se han encontrado en zonas rodeadas de creta.

Con el tiempo para conseguir los materiales era necesario hacer excavaciones para conseguir el material, y así se conformaron las primeras minas, cuya constancia arqueológica de la más antigua data de hace 43 mil años, como es la Cueva de León en Suazilandia en el África Austral o del Sur.

En el continente americano, igualmente se encontraron minas muy antiguas, y con el transcurrir del tiempo esta actividad extractiva cobró gran importancia para la economía de los países.

Con el transcurrir del tiempo se ha demostrado que la extracción minera siempre ha sido una actividad con un alto índice de accidentabilidad, de alto riesgo; por lo que las autoridades reglamentaron las condiciones de trabajo y establecieron las normas de seguridad y salud en las organizaciones (Laitinen \& Ruohomäki, 1996).

En nuestro país, la extracción minera se encuentra reglamentada por diversa normativa, así como se crearon organismos supervisores de seguridad laboral como la Osinergmin y la SUNAFIL; y se obliga a las empresas mineras a proveer a sus trabajadores de Equipos de Protección Personal EPP, para su seguridad; así como la conformación de comités de seguridad dentro de los centros laborales.

Así también, no obstante la normativa y políticas de seguridad, los accidentes en la actividad minera siguen sucediendo, ya sea por la utilización de máquinas de alta peligrosidad y de difícil maniobrabilidad para el trabajador, y por el ambiente en que se desarrolla la extracción de materiales, ya sea a tajo abierto y minería subterránea o con socavones, donde el riesgo al peligro por naturaleza está presente (Hickman \& Geller, 2003).

Cabe mencionar también, que el Ministerio de Energía y Minas (MEM, 2015) publica cada año la relación de los accidentes graves que se producen cada año en las empresas mineras, como evidencia de la importancia que se le viene dando a la seguridad en la actividad minera (Becerril, 2013).

\section{METODOS}

\subsection{Tipo y diseño de investigación}

La presente investigación trata de determinar el grado de relación entre las variables planteadas aplicando el diseño correlacional entre la aplicación de la intervención de los colaboradores teniendo una relación directa para reducir el índice de accidentabilidad en la empresa contratista minera AESA (Alvarez, 2015).
El diagrama esquemático del diseño es:

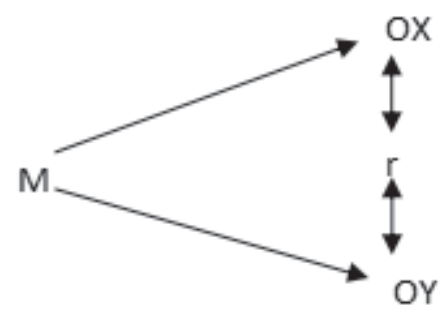

Donde:

$\mathrm{M}=$ muestra de estudio

$\mathrm{Ox}=$ Evaluación $\mathrm{y} / \mathrm{u}$ observación de la variable: Aplicación del método de intervención.

$\mathrm{r}=$ Relación entre variables, Aplicación del método de intervención y reducción del índice de accidentabilidad (Díaz Vega \& Rodríguez Bobadilla, 2010).

Oy = Evaluación y/u observación de la variable: Índice de accidentabilidad.

\subsection{Unidad de análisis}

La unidad de análisis es la empresa contratista minera AESA.

\subsection{Población de estudio}

Para efectos del presente estudio se considera a toda la población que brinda servicios operativos en la empresa contratista minera AESA. Contando con un total de 280 colaboradores entre personal empleado y obrero. El estudio se realiza al personal Operativo. Conformándolo de la siguiente manera: Supervisores estratégicos.- Residente de obra, Jefe de Operaciones, Ingeniero de Seguridad, Supervisores de línea.-Jefes de guardia, supervisores técnicos. Personal Operativo.- Personal de operación de equipos de perforación (Jumbos), personal de operación de equipos de limpieza (Scoop), Ayudante de operador de Jumbo, personal de carguío y voladura y mecánicos de jumbo-scoop, personal de servicios auxiliares.

Dentro de la muestra seleccionada estadísticamente se considera a un total de 24 colaboradores (Ver Tabla 1).

\section{RESULTADOS}

\subsection{Resultados obtenidos de la recolección de datos proporcionados por la empresa}

En la figura 1 se observa que el 91\% de los trabajadores tiene actos inseguros relacionados al orden y limpieza de su área de trabajo, el 81\% tiene falta de concentración en el trabajo que está realizando, el $70 \%$ de los trabajadores no emplean todos sus implementes EPPs, el 67\% no hace el uso correcto de equipos y herramientas de trabajo (Montero Martinez, 2003), el mismo porcentaje de trabajadores no emplean el IPERC, PETAR y otras herramientas para le prevención de riesgos, el $66 \%$ de los trabajadores no cumplen con los procedimientos, normas e instructivos de trabajo, el $29 \%$ se expone a movimientos repetitivos y situaciones incómodas al momento de trabajar, el $27 \%$ 
de los trabajadores tiene un ritmo de trabajo inadecuado, finalmente el $18 \%$ de los trabajadores no se comunica de forma adecuada con su compañeros ni supervisores (Bellone, 2008).

En la figura 2 tenemos que el $82 \%$ de los trabajadores tiene una buena comunicación con sus compañeros y supervisores, el $73 \%$ tiene un ritmo de trabajo adecuado con sus labores que realiza, el $71 \%$ evita movimientos o posiciones incómodas al realizar sus labores, sin embargo solo el 34\% cumplen con los procedimientos, normas e instructivos de trabajos, así mismo el 33\% hace uso de IPERC, PETAR y otras herramientas para la prevención de accidentes, el $33 \%$ de los trabajadores hace uso correcto de equipos herramientas de trabajo (Candia, Renan \& T Hennies, W \& C Azevedo, R \& G Almeida, I \& F Soto, 2018), el 30\% de trabajadores emplea sus EPPs adecuadamente, el 19\% tiene buena concentración en las labores que realiza, finalmente tan solo el $9 \%$ de los trabajadores tiene su área de trabajo limpio y ordenado.

Según la figura 3 indica que el acto inseguro con menor porcentaje es la comunicación con sus compañeros de trabajo y sus supervisores $(18 \%)$, el problema del ritmo de trabajo que existe no es el adecuado (27\%), luego se observa que los movimientos inseguras en el área de

Tabla1. Distribución de la muestra por puestos de trabajo

\begin{tabular}{lccc}
\hline Puesto de trabajo & Condición & Total & Muestra \\
\hline Jefe de operaciones & Empleado & 3 & 0 \\
Ingeniero seguridad & Empleado & 4 & 0 \\
Supervisor de operaciones & Empleado & 18 & 0 \\
Supervisor de seguridad & Empleado & 3 & 0 \\
Personal operador de jumbo & Obrero & 21 & 6 \\
Personal ayudante de operador de jumbo & Obrero & 21 & 3 \\
Personal operador de Scoop & Obrero & 24 & 3 \\
Personal de carguío y voladura & Obrero & 24 & 6 \\
Personal de servicios auxiliares & Obrero & 12 & 3 \\
Mecánicos de equipo jumbos y scoop & Obrero & 15 & 3 \\
\hline
\end{tabular}

Fuente: Elaboración propia

Tabla 2. Reporte de intervenciones del personal de seguridad

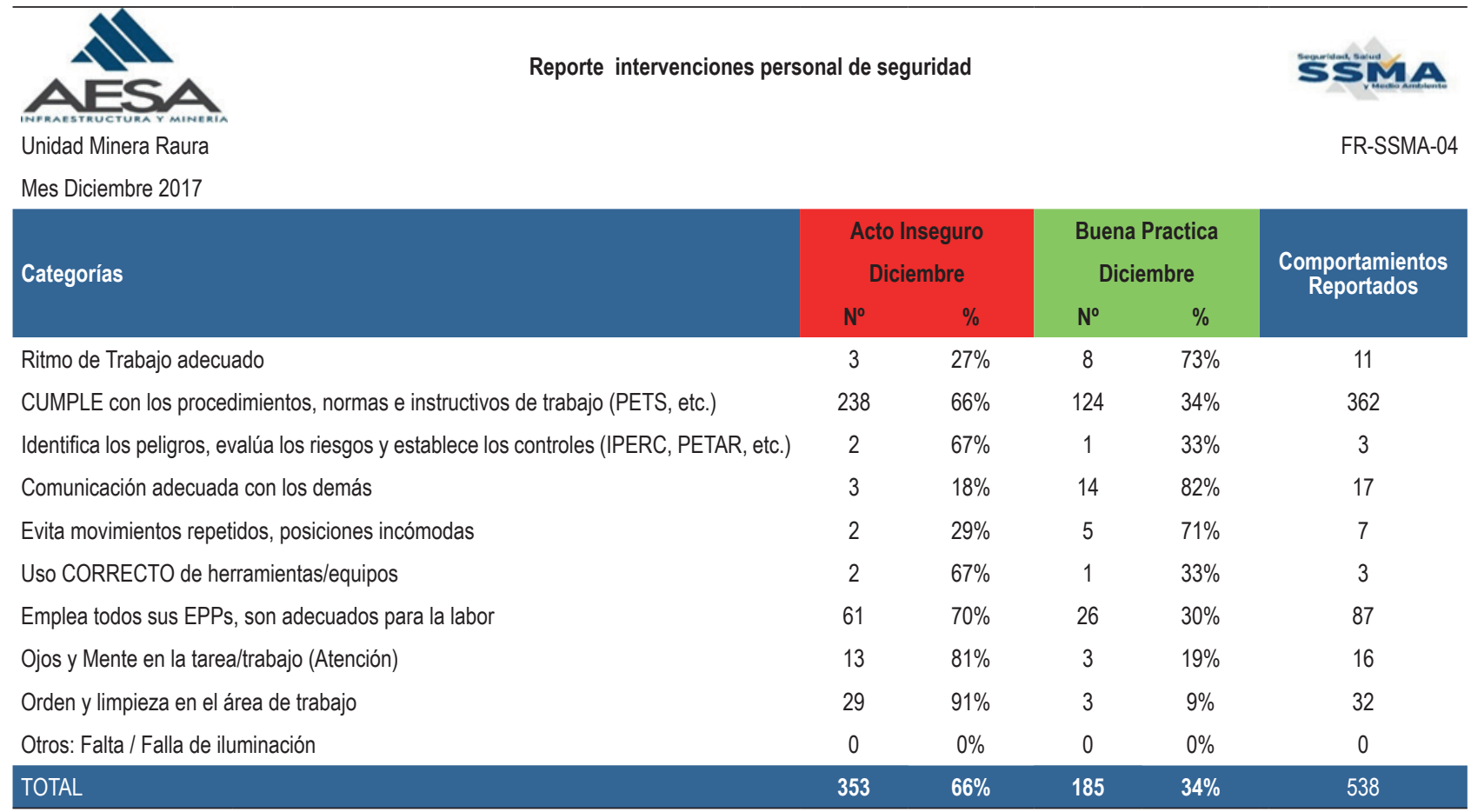

Fuente: Minera Raura (2020) 


\section{Resumen actos inseguros}

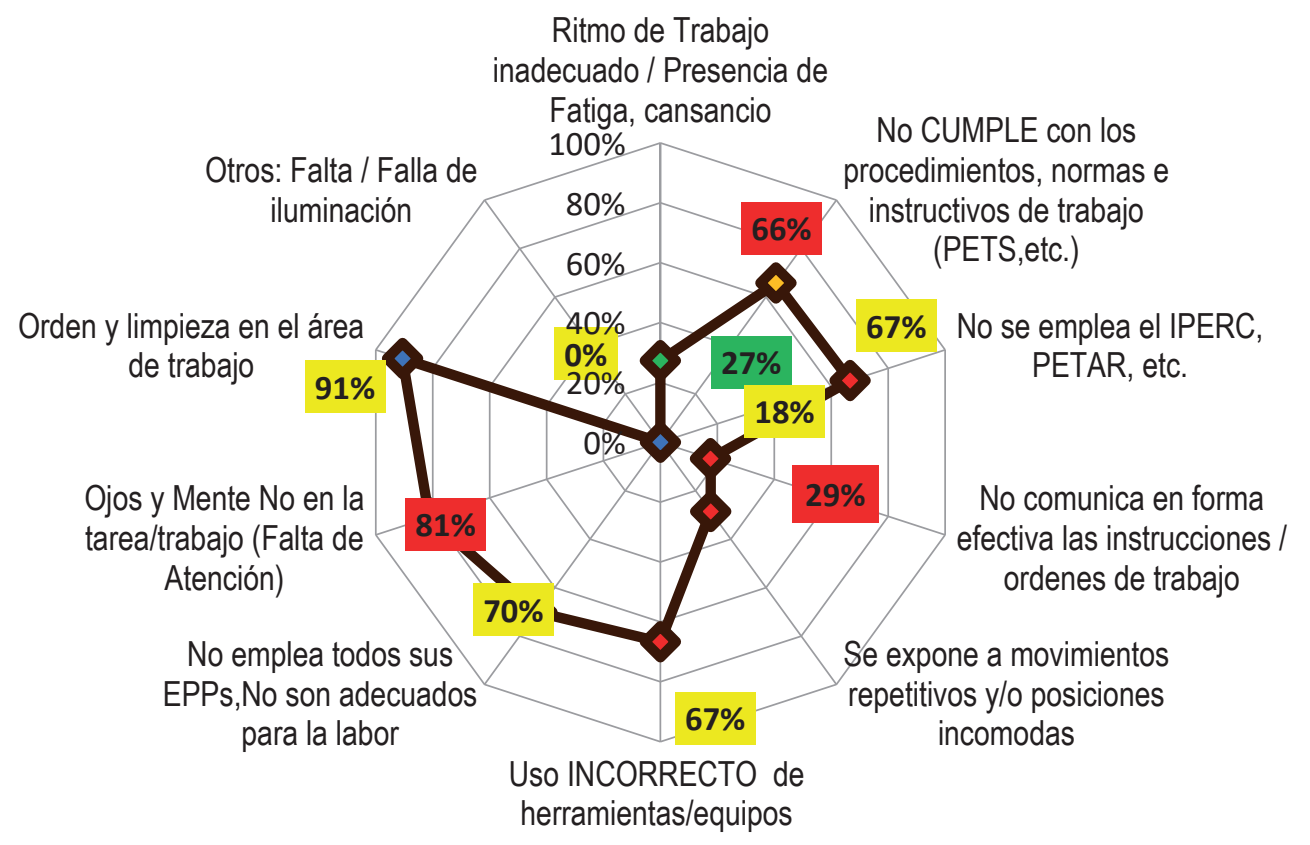

Figura 1. Actos inseguros. Fuente: Minera Raura (2020)

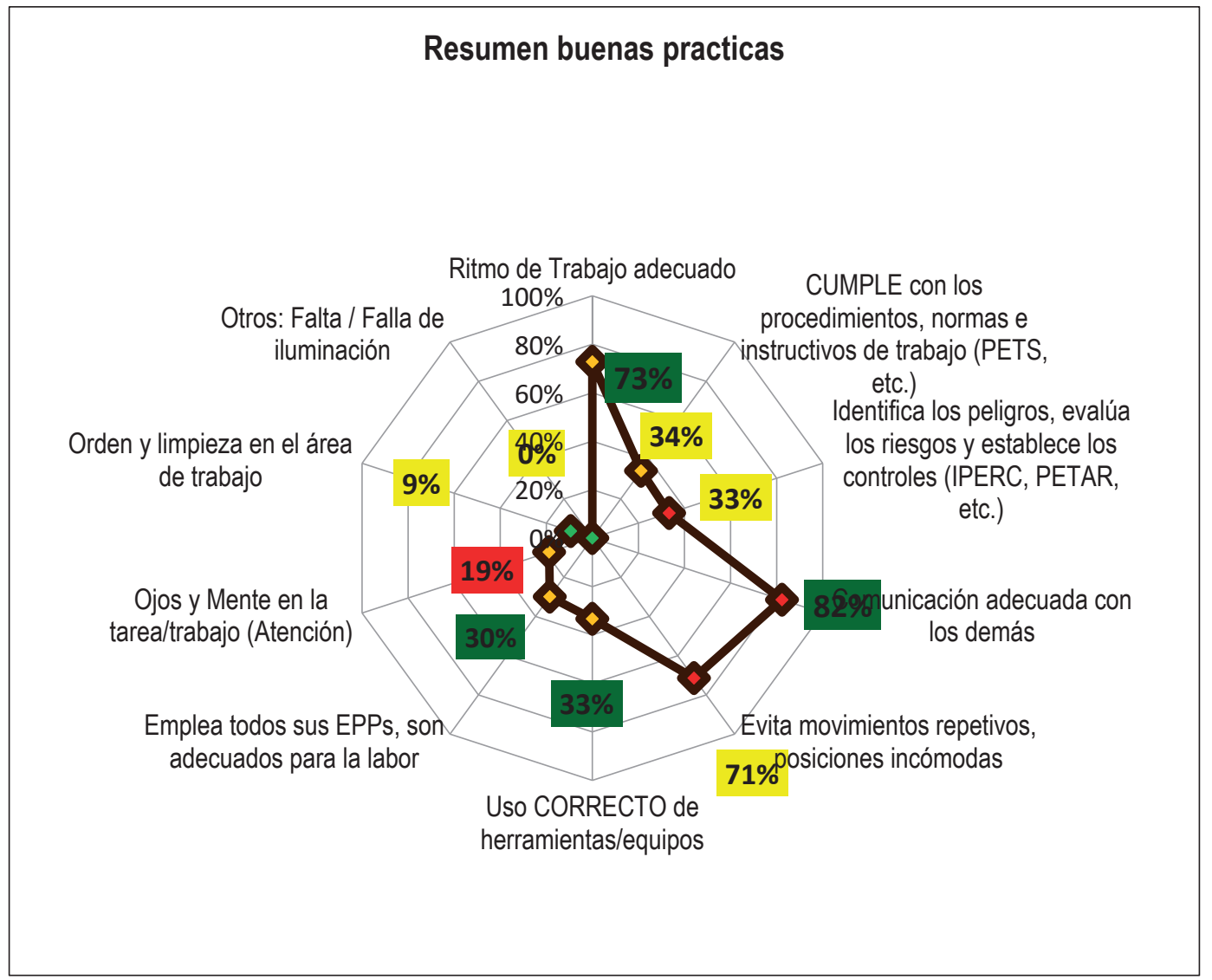

Figura 2. Resumen de buenas prácticas. Fuente: Minera Raura (2020) 
trabajo es otro factor para decir que es un comportamiento inseguro (29\%) (Geller et al., 1997) de este acto se ve que el porcentaje aumenta considerablemente hasta el $66 \%$ de los trabajadores quienes no siguen los procedimientos y normativas de trabajo, pero el que llama más la atención es que el $91 \%$ de los trabajadores tienen su área de trabajo desordenada y que conlleva a un comportamiento inseguro que es un peligro constante para sufrir algún accidente (Fox et al., 1987).

\section{RESULTADOS}

De acuerdo a la figura 4 se observa que el mes de mayo hubo un 3.22\% de accidentes incapacitantes (Meliá Navarro, 1995), luego en el mes de junio se registraron 5.36\% de estos accidentes. A partir de julio empezó una tendencia a disminuir el porcentaje de accidentes incapacitantes, habiéndose registrado el mes de diciembre un $2.43 \%$ de accidentes incapacitantes (Meliá, 2007).

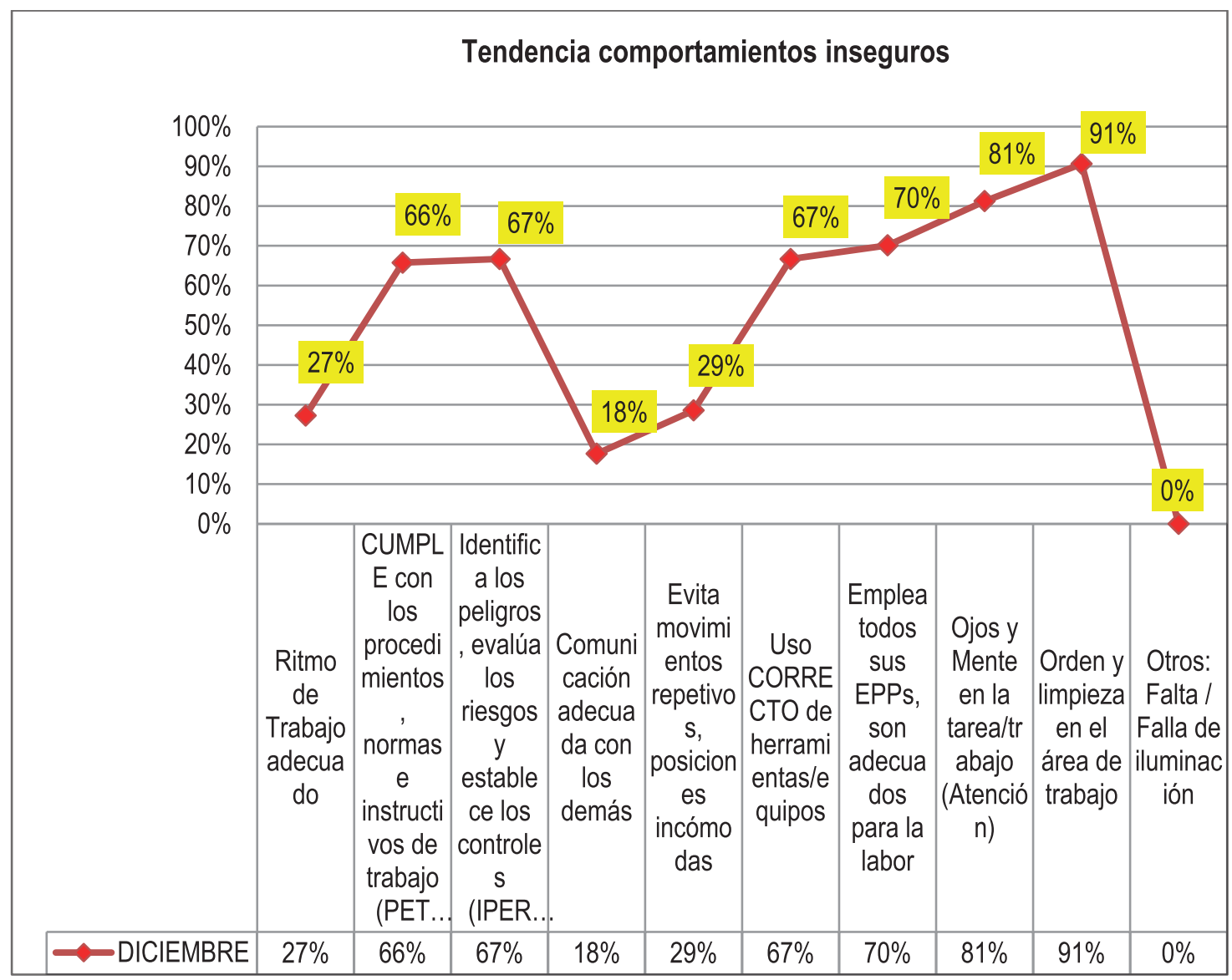

Figura 3. Porcentajes de comportamientos inseguros. Fuente: Minera Raura (2020)

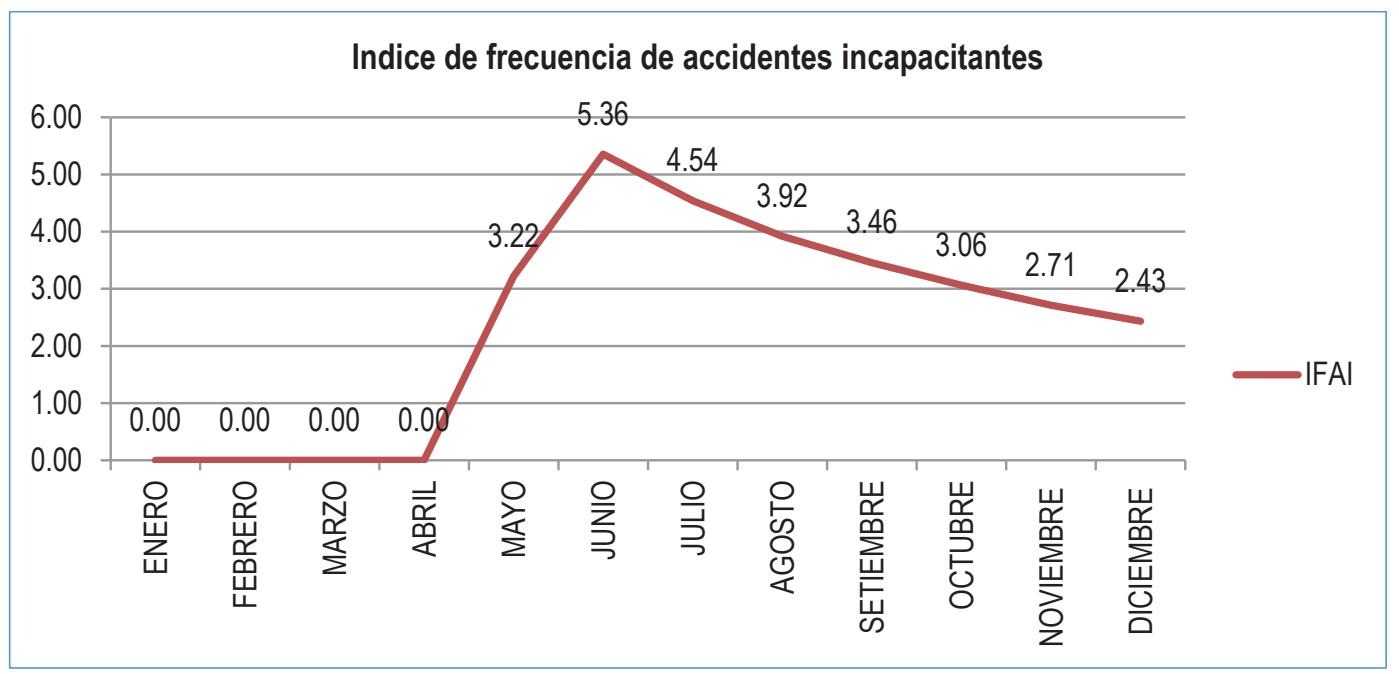

Figura 4. Accidentes incapacitantes. Fuente: Elaboración propia 
De acuerdo a la figura 5 se observa que en el mes de abril se registraron $4.05 \%$ de lesiones, en el mes siguiente se registró un $6.44 \%$ teniendo un incremento hasta el mes de julio con $19.8 \%$ de lesiones, a partir del mes de agosto $(7.84 \%)$ las lesiones se desaceleraron hasta el mes de octubre $(6.12 \%)$, y el mes de noviembre la frecuencia de lesiones aumentó a $6.78 \%$, finalmente en el mes de diciembre se registró una leve baja en el porcentaje de lesiones registradas $(7.30 \%)$.

\section{CONCLUSIONES}

1. La influencia del método de intervención en la reducción del índice de accidentabilidad en la empresa contratista minera AESA ha sido altamente positiva puesto que los trabajadores se han informado, capacitado y concientizado en los temas de seguridad mediante la implementación de este método.

2. La influencia del método intervención en la mejora de la cultura de seguridad en una contratista minera AESA ha sido considerablemente positiva puesto que antes de haber aplicado el método solo el $25 \%$ de los participantes dijo que sí sabía qué es seguridad laboral y el 75\% dijeron que no sabían, sin embargo después de aplicar el método de intervención el $87.5 \%$ dijo que sí sabía que era seguridad laboral, estos datos indican que los trabajadores ahora se tienen más conocimientos sobre la reducción y prevención de accidentes en el área laboral.

3. La influencia del método intervención en la reducción del porcentaje rotación del personal en una contratista minera es considerablemente positiva puesto que los trabajadores antes de aplicar el método de intervención se sentían inseguros o poco seguros al no saber métodos para la disminución y prevención de los accidentes, no sabían los beneficios de tener un seguro de vida, para mantenerse más seguros en su lugar de trabajo y evitar la rotación del personal.
4. Finalmente, la influencia del método de intervención en la reducción de la frecuencia de accidentes incapacitantes en una contratista minera ha sido considerablemente puesto que los trabajadores han sido capacitados mediante el método sobre los beneficios que ofrece el IPERC para calificarlos según la peligrosidad que representa alguna actividad en su área de trabajo (Milla Lliuya, 2013), permitiendo de esta forma guardar las medidas del caso y prevenir accidentes que lleven a la incapacidad de algún trabajador (Guastello, 1993).

\section{AGRADECIMIENTOS}

Al Ing. Harold Deza Guzmán, Ingeniero de materiales de la Universidad Nacional de Trujillo

\section{REFERENCIAS}

Alvarez, A. (2015). Implementación del programa de comportamiento seguro y evaluación de resultados como técnicas de intervención efectiva para reducir la accidentabilidad en mina San Juan de Chorunga. http:// bibliotecas.unsa.edu.pe/handle/UNSA/161

Becerril, M. (2013). Un proceso de intervención sobre las conductas de seguridad y las condiciones de seguridad y salud en las obras de construcción. http://mobiroderic. uv.es/bitstream/handle/10550/29248/Tesis M Becerril. pdf? sequence $=1 \&$ isAllowed $=y$

Bellone, G. J. (2008). La Psicología de la Seguridad como herramienta preventiva en la industria minera: Un estudio de Caso en Minera Alumbrera Argentina - Xstrata Copper. http://sistemasreid.com.ar/wp-content/uploads/2017/03/ Congreso-ORP-Chile-2013-Póster-La-Psicología-de-laSeguridad-como-herramienta-preventiva-en-la-IndustriaMinera.pdf

Candia, Renan \& T Hennies, W \& C Azevedo, R \& G Almeida, I \& F Soto, J. (2018). Notificación de accidentes mortales en el sector de minería peruana 2000-2014. Insight Medical Publishing. https://www.archivosdemedicina.com/

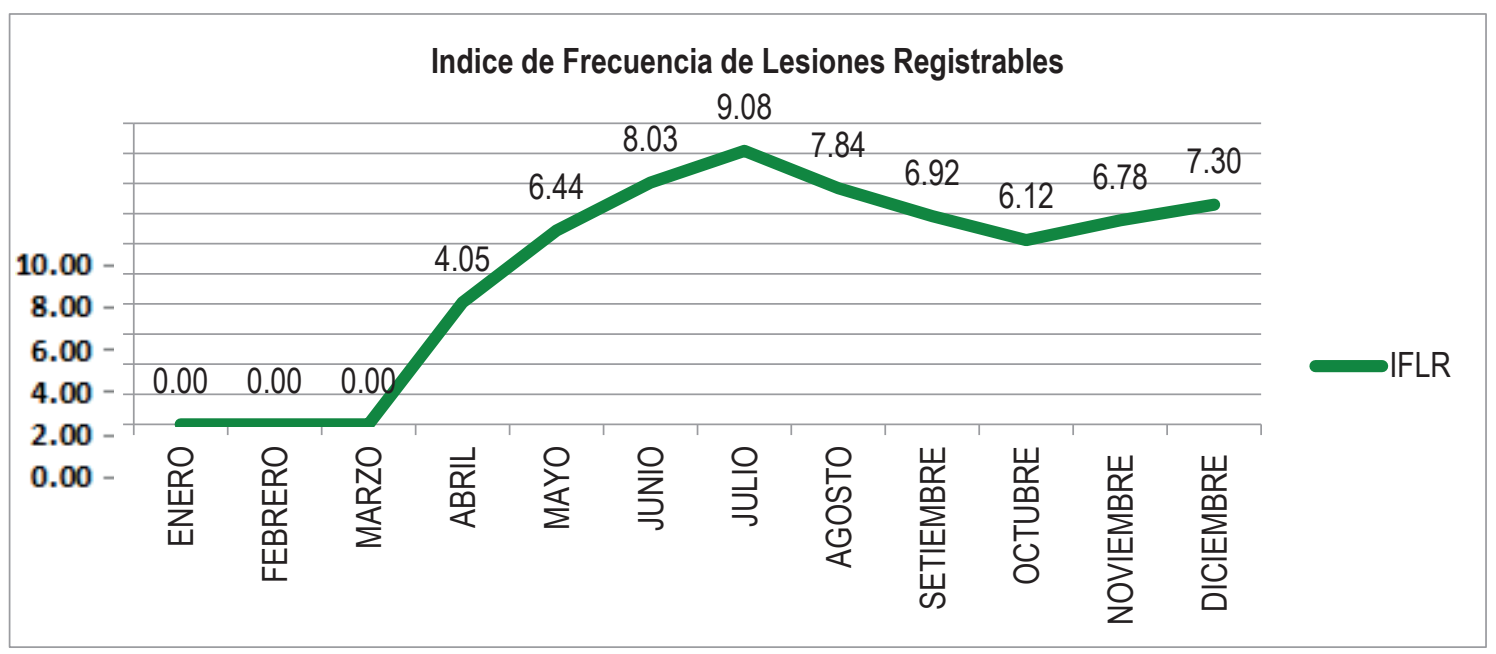

Figura 5. Lesiones registrables. Fuente: Elaboración propia 
medicina-de-familia/notificacin-de-accidentes-mortalesenel-sector-de-minera-peruana-20002014accidentesmortales-en-minera-peruana.php?aid $=8058$

Díaz Vega, J. A., \& Rodríguez Bobadilla, J. L. (2010) Implementación de un sistema de gestión de Seguridad y Salud ocupacional para la reducción de accidentes en la UEA Sector Arequipa 2015. In Universidad Privada del Norte. https://repositorio.upn.edu.pe/handle/11537/23916

Fox, D. K., Hopkins, B. L., \& Anger, W. K. (1987). The long-term effects of a token economy on safety performance in openpit mining. Journal of Applied Behavior Analysis, 20(3), 215-224. https://doi.org/10.1901/jaba.1987.20-215

Geller, E. S., Carter, N., Depasquale, J., Pettlnger, C. \& Wllllams, J. (1997). Applications of Behavioral Science to Improve Mine Safety. Concepts and Processes in Mine Health and Safety Management, Society for Mining, Metallurgy, and Exploration, Inc., 65-81. http://citeseerx.ist.psu.edu/viewdoc/ download?doi=10.1.1.466.7080\&rep=rep1\&type $=$ pdf

Guastello, S. J. (1993). Do we really know how well our occupational accident prevention programs work? Safety Science, 16(3-4), 445-463. https://doi.org/10.1016/09257535(93)90064-K

Hickman, J. S., \& Geller, E. S. (2003). A safety self-management intervention for mining operations. Journal of Safety Research, 34(3), 299-308. https://doi.org/10.1016/S00224375(03)00032-X

Laitinen, H., \& Ruohomäki, I. (1996). The effects of feedback and goal setting on safety performance at two construction sites. Safety Science, 24(1), 61-73. https://doi.org/10.1016/

\section{S0925-7535(96)00070-7}

Meliá, J. L. (2007). Seguridad basada en el comportamiento. Universidad de Valencia, 157-180. https://www. uv.es/ meliaj1/Papers/2007JLM_SBC.pdf

Meliá Navarro, J. (1995). Un proceso de intervención para reducir los accidentes laborales. Revista de Psicología Del Trabajo y de Las Organizaciones = Journal of Work and Organizational Psychology, 11(32), 51-66. https://dialnet. unirioja.es/servlet/articulo?codigo $=109518$

Milla Lliuya, O. D. (2013). Evaluación del nivel de gestión de riesgos para la mejora continua de la seguridad y salud en el proceso de minado Marañón CIA. Minera Poderosa S.A. In Universidad Nacional de Ingeniería. http://cybertesis.uni. edu.pe/handle/uni/3760

Minera Raura. (2020). Compañía Minera Raura S.A. Anddes, Asociados. http://anddes.com/proyecto/raura/

Ministerio de Energía y Minas -MEM. (2015). Fax Coyuntural de accidentes mortales año 2015. Dirección de Técnica Minera. http://www.minem.gob.pe/_estadistica. php?idSector $=1 \&$ idEstadistica $=12464$

Montero Martinez, R. (2003). Siete principios básicos de la Seguridad basada en los comportamientos. Prevención, Trabajo y Salud: Revista Del Instituto Nacional de Seguridad e Higiene En El Trabajo, 25, 4-11. https://dialnet.unirioja. es/servlet/articulo? codigo $=1012049$ 
